\title{
Reflets
}

Revue ontaroise d'intervention sociale et communautaire

\section{Présentation - Reflets : revue ontaroise d'intervention sociale et communautaire}

\section{Richard Carrière}

Volume 1, numéro 1, printemps 1995

Des pratiques à notre image : défis et ressources

URI : https://id.erudit.org/iderudit/026050ar

DOI : https://doi.org/10.7202/026050ar

Aller au sommaire du numéro

Éditeur(s)

Reflets : Revue ontaroise d'intervention sociale et communautaire

ISSN

1203-4576 (imprimé)

1712-8498 (numérique)

Découvrir la revue

Citer ce document

Carrière, R. (1995). Présentation - Reflets : revue ontaroise d'intervention sociale et communautaire. Reflets, 1(1), 1-8. https://doi.org/10.7202/026050ar

Tous droits réservés (C) Reflets : Revue ontaroise d'intervention sociale et communautaire, 1995
Ce document est protégé par la loi sur le droit d'auteur. L'utilisation des services d'Érudit (y compris la reproduction) est assujettie à sa politique d'utilisation que vous pouvez consulter en ligne.

https://apropos.erudit.org/fr/usagers/politique-dutilisation/ 


\section{Présentation}

\section{Reflets: revue ontaroise $d^{\prime}$ 'intervention sociale et communautaire}

N ous sommes heureux de vous offrir ce premier numéro de $R$ eflets: revue ontaroise d'intervention sociale et communautaire. Plusieurs lecteurs et lectrices ont sans doute participé de près ou de loin au processus de mise sur pied de cette nouvelle revue. $D$ ans cette brève présentation, nous aimerions retracer les grandes lignes de son développement et présenter sa raison d'être ainsi que quelques informations sur son contenu.

\section{Les débuts de la revue...}

L'idée de créer une revue ontaroise a germé en 1992 et fait son chemin depuis ce temps, se concrétisant lentement grâce à l'apport et à l'encouragement de nombreuses personnes. Le 29 octo bre 1992, une vingtaine de personnes représentant divers milieux de la pratique et diverses régions de la province se sont regroupées à l'École de service social de l'U niversité L aurentienne pour discuter des défis reliés à la mise sur pied d'une telle revue.Y ves Vaillancourt, membre fondateur de la revue québécoise $N$ ouvelles pratiques sociales et professeur en service social à l'U niversité du Q uébec à $M$ ontréal a été invité à cette rencontre pour partager son expérience et susciter une réflexion plus approfondie sur les enjeux d'une telle initiative.

Suite à cette première rencontre, soit en février 1993, un sondage a été effectué auprès de 734 intervenantes et intervenants 
francophones de l'O ntario afin d'évaluer l'intérêt suscité par la revue proposée. C e sondage a été subventionné par le Comité francophone de l'A ssociation des travailleuses et des travailleurs sociaux professionnels de l'O ntario (ATSPO). D eux cent cinquantesept personnes ont répondu au questionnaire, soit un taux de réponses de $35 \%$. C es réponses indiquaient que $99 \%$ des personnes étaient intéressées à lire la revue; $90 \%$ se sont montré intéressées à s'y abonner et $44 \%$ ont indiqué qu'elles seraient prêtes à soumettre des articles.

Encouragé par cet intérêt de la communauté francoontarienne, le groupe organisateur du projet organise une deuxième rencontre, le 18 novembre 1993, à l'École de service social de l'U niversité d'O ttawa, afin de discuter de façon plus concrète la mise sur pied de la revue.

Un comité d'implantation, composé de $\mathrm{R}$ ichard $\mathrm{C}$ arrière (Sudbury), R ichard M artel (Toronto), H élène Gagné (Toronto), $M$ ichèle Kérisit (O ttawa), C hris Sassa (Toronto), C éline Simard (Sturgeon Falls) et N érée St-A mand ( 0 ttawa) est alors mis sur pied. Dès le départ, le comité s'entend pour privilégier un partenariat entre les milieux universitaire et communautaire. $Q$ uelques semaines plus tard, $M$ adeleine $D$ ubois ( 0 ttawa) se joignait au comité. Elle est, avec N érée St-Amand, co-rédactrice de ce premier numéro.

C e comité de bénévoles engagés se met à l'oeuvre sans tarder. D e nombreuses tâches et décisions s'imposent: définir la mission de la revue, identifier les thèmes des premiers numéros, choisir les diverses rubriques, lancer des appels de communication, sélectionner une maison d'édition, trouver des sources de financement, choisir un titre approprié, etc. Grâce à l'appui financier du ministère des Services sociaux et communautaires et du ministère de la Santé, les membres du comité ont accèsà certaines ressources ou consultations pour faciliter l'exécution des multiplestâches qui leur incombent. 


\section{Pourquoi une revue ontaroise sur l'intervention sociale et communautaire?}

Tout d'abord pour combler une lacune évidente... En 0 ntario, les intervenantes et intervenants qui oeuvrent auprès de la communauté francophone dans le domaine des pratiques sociales et communautaires n'ont aucun outil de communication qui leur soit propre. La revue permettra de découvrir, d'analyser et de remettre en question l'évolution, la définition et la distribution des programmes et des services d'intervention sociale, communautaire et de santé en 0 ntario français.

$R$ eflets veut aussi promouvoir la réflexion inter et multidisciplinaire sur le développement et les pratiques sociales, communautaires et de santé, sur les politiques sociales et leurs enjeux et sur la formation professionnelle. Plusieurs personnes qui travaillent actuellement avec la population franco- ontarienne tentent d'approfondir les réflexions et l'analyse portant sur les spécificités de leurs pratiques; nous nous proposons, par l'entremise de cette revue, d'identifier et d'explorer plus à fond lescaractéristiques faisant en sorte que l'intervention en 0 ntario français ne puisse pas reproduire ce qui se passe ailleurs, en raison des particularités historico-socio-politiques en contexte minoritaire. $\mathrm{N}$ ous voulons faciliter un forum d'échanges et offrir aux intervenantes et aux intervenants un débouché pour diffuser le résultat de leurs recherches, faire part de réflexions sur leur champ de pratique et d'analyses critiques des politiques sociales et de leurs répercussions sur la population franco- ontarienne.

Les programmes sociaux et communautaires de notre province ont des particularités, des orientations et une histoire qui les rendent uniques. De plus, plusieurs programmes et services connaissent actuellement des transformations majeures.Tout ceci soulève des questions pour nos pratiques qui doivent être débattues et analysées.

$D$ ans le contexte de tous ces changements, les intervenantes et intervenants ont aussi besoin d'une formation professionnelle 
de qualité. À cet effet, R eflets compte publier des textes et des réflexions pour stimuler, appuyer ou illustrer le contenu de présentations professionnelles. Présentement, nous devons, la plupart du temps, avoir recours à des textes provenant de l'extérieur de la province ou du pays et qui ne correspondent pas nécessairement à certains de nos besoins spécifiques.

En somme, le mandat de $R$ eflets est de partager l'information et de stimuler la réflexion des intervenantes et des intervenants francophones sur les diverses pratiques sociales et communautaires en 0 ntario.

\section{Pour qui?}

Pour vous et vos collègues des quatre coins de la province. La revue s'intéresse à l'ensemble des personnes engagées dans la promotion du bien-être de leur communauté, qui doivent renouveler leurs pratiques par la recherche de solutions réfléchies et novatrices. N ous voulons que cette revue soit un lieu d'échanges entre les intervenantes et les intervenants qui travaillent directement et indirectement auprès de la population franco-ontarienne ainsi qu'entre les personnes impliquées dans la formation collégiale ou universitaire de nos futurs intervenants et intervenantes francophones. R eflets cherche aussi à rejoindre celles et ceux qui oeuvrent bénévolement dans la multitude d'organismes communautaires, aussi bien dans les secteurs de la santé communautaire et des services sociaux que dans les secteurs correctionnels, éducationnels et de santé.

B ref, la revue s'adresse à vous si votre pratique porte sur la promotion du mieux-être de la personne et de la communauté, sur la prévention des problèmes sociaux et de santé ainsi que sur l'amélioration des conditions de vie de la population francoontarienne.

Les rubriques de la revue ont été planifiées pour donner à toutes et tous une place pour s'exprimer. Voici une brève description de ces rubriques. 


\section{Entrevue/table-ronde}

C ette rubrique a pour but de vous «brancher» sur un événement ou une problématique d'actualité par l'entremise d'un échange avec une ou des personnes engagées dans le milieu. $\mathrm{N}$ ous nous ferons un plaisir d'organiser des entrevues avec des personnes qui oeuvrent partout en province et nous comptons sur nos lecteurs et lectrices pour suggérer des personnes ou sujets à présenter par l'entremise de cette rubrique.

\section{Le dossier}

Le dossier regroupe des articles de fond qui ont été sollicités par un appel de communications. C es articless'articulent autour d'un thème et cette rubrique consacrera donc une place importante à l'analyse d'un domaine spécifique. $D$ ans ce premier numéro, le dossier porte sur «D es pratiques socio-communautaires à notre image» et comprend six articles. $D$ ans le deuxième numéro, D ossier se penchera sur la santé communautaire, alors que dans les numéros subséquents, on y abordera l'intervention pour contrer la violence au sein de nos communautés, l'intervention auprès des ânées et aînés et auprès des personnes ayant des besoins spéciaux.

La sélection des thèmes est faite par les membres du comité de rédaction composé de dix-sept personnes des quatre coins de la province. Le nom de ces membres figure d'ailleurs à la page iii de la revue. Si vous avez des suggestions pour la rubrique D ossier, n'hésitez pasà nous les transmettre ou à en discuter avec les membres du comité de rédaction. II est important de souligner que les articles prévus pour la rubrique $D$ ossier sont arbitrés anonymement par un comité de lecture, afin d'assurer leur qualité et leur pertinence.

\section{Des pratiques à notre image}

C ette rubrique nous permet d'inclure des articles plus brefs que ceux du dossier, mais qui présentent des pratiques sociales 
et communautaires en 0 ntario, dans une perspective historique, analytique ou autre Ces articles ne sont pas soumis à l'arbitrage extérieur, maissont revus par l'équipe responsable de chaque numéro.

\section{Aux quatre coins de la province}

Dans cette rubrique, vous êtes invités à partager les nouvelles brèves, les comptes rendus d'expériences et de mise sur pied de programmes dansvos régions. Elle présentera aussi des reportages sur les ateliers, les conférences, les outils de formation qui ont été utiles.

\section{Aux études}

Voici une rubrique vous familiarisant avec le contenu des mémoires et des thèses des étudiantes et étudiants terminant leurs études de deuxième ou troisième cycle dans un domaine lié aux objectifs ou à la mission de R eflets.

\section{Lu pour vous}

C ette rubrique présente des comptes rendus de ressources (livres, rapports, vidéos, etc.) pertinentes à l'intervention en français en O ntario.

\section{Une revue faite par qui?}

Par nous (une équipe dynamique) et par vous! Comme vous pouvez le constater par l'organisation des rubriques, cette revue est un projet communautaire qui exige votre participation.Votre abonnement à la revue et votre participation comme auteurs et auteures assureront le succès du projet. $N$ ous vous invitons à vous joindre à nous...

$\mathrm{N}$ ous sommes conscients des défis inhérents à cette invitation. Vous êtes sans doute débordés par votre travail, par vos engagements multiples et pour plusieurs, l'idée d'être confronté à l'écriture 
représente un défi de taille. M ais plusieurs personnes l'ont déjà fait dans ce premier numéro, et toutes et tous sont satiffaits de l'expérience.

En 0 ntario français, nousn'avons pasl'habitude d'écrire! Q ue ce soit avec la plume ou avec l'ordinateur, nous hésitons a mettre sur papier nos idées, nos expériences, nos réflexions. La tradition orale a des assises plusgrandesquel'écrit dansnotre culture francoontarienne. $N$ ous croyons cependant que vous êtes en mesure de relever ce défi. L'équipe de rédaction est là pour vous appuyer. C'est ensemble qu'on réussira à se donner un outil de partage qui nous permettra de faire avancer les dossiers qui nous tiennent à coeur.

\section{Remerciements}

En terminant cette présentation, nous aimerions souligner la contribution de plusieurs personnes et organismes qui ont appuyé les démarches de mise sur pied de $R$ eflets.

D'abord, merci aux écoles de service social de I'U niversité Laurentienne et de I'U niversité d' $O$ ttawa ainsi qu'au C entre ontarien d'information en prévention et au Service familial de Sudbury qui, dès le départ, nous ont appuyés en permettant à certains membres de leur personnel de s'engager dans la planification de la revue. Ces quatre organismes ont aussi offert leurs locaux pour les rencontres des comités de rédaction et de direction. Leur collaboration est très appréciée.

M erci au comité francophone de l'A ssociation des travailleuses sociales et des travailleurs sociaux professionnels de l'O ntario pour leur appui financier et leur encouragement dans nos démarches.

$N$ ous aimerions aussi remercier $M$. R éjean $N$ adeau, coordonnateur des services en langue française au ministère des Services sociaux et communautaires, M. Gilles H uot, coordonnateur des services en langue française au ministère de la Santé ainsi que $M$ de Suzette Bédard-Philippe, coordonnatrice des servicesen français au ministère du Solliciteur général et des Services correctionnels, 
pour leur précieux appui. C 'est grâce à l'appui financier de leurs ministères respectifs que nous sommes en mesure de vous offrir ce premier numéro de la revue.

$\mathrm{N}$ ous tenons à remercier chaleureusement la co-rédactrice et le co-rédacteur de ce premier numéro, $M$ adeleine $D$ uboiset $N$ érée St-A mand. A ppuyées de Sonia $C$ adieux et de $M$ ichèle K érisit, ces deux personnes ont consacré d'innombrables heures à la préparation de ce premier numéro dont nous sommes très fiers.

Enfin, un remerciement tout spécial aux intervenantes et intervenants, tant communautai res qu'univer sitai resqui ont accepté de relever le défi d'écrire et de partager leurs communications. Un gros merci !!!

Sur ce, bonne lecture.

$\mathrm{R}$ ichard $\mathrm{C}$ arrière

Au nom du comité de direction de $R$ eflets 